\title{
Electrochemical Synthesis of Reduced Graphene Oxide-Wrapped Polyaniline Nanorods As An Active Nanocomposite For Improved Photocurrent Generation And Photocatalytic And Antibacterial Activities
}

\section{Fares Fenniche}

University of Kasdi Merbah

Abdellah Henni ( $\nabla$ henni.abdellah@gmail.com )

University of Kasdi Merbah https://orcid.org/0000-0002-5054-5442

Yasmine Khane

University of Ghardaïa

Djaber Aouf

University of Kasdi Merbah

Nessrine Harfouche

Université du Sud Toulon

Soufiane Bensalem

Kasdi Merbah University

Djamal Zerrouki

University of Kasdi Merbah

Hakim Belkhalfa

Scientific and Technical Research Center in Physicochemical Analysis

\section{Research Article}

Keywords: Polyaniline nanorods, Reduced graphene oxide, Organic nanocomposite, Photocurrent, Photocatalytic activity.

Posted Date: October 1st, 2021

DOI: https://doi.org/10.21203/rs.3.rs-946061/v1

License: (c) (i) This work is licensed under a Creative Commons Attribution 4.0 International License.

Read Full License 


\section{Abstract}

This study depicts the electrochemical synthesis of nanocomposites basede on Polyaniline nanorods wrap with reduced graphene oxide (PANI-rGO) on ITO substrates. Synthesis of PANI-rGO nanocomposites was elaborated by the incorporation of rGO in PANI thin films during electropolymerization in the presence of sulfuric acid. The synthesis of reduced graphene oxide was by modification on the wellknown Hammer's method. The thin films nanocomposites were characterized by X-ray photoelectron spectroscopy (XPS), Scanning electron microscopy (FESEM), UV-Visible and electrochemical photocurrent spectroscopy. FESEM revealed the formation of PANI nanorods with diameters between 50 and $150 \mathrm{~nm}$. The XPS was employed to confirm the compositions of PANI-rGO nanocomposites. From photoelectrochemical results, the generated photocurrent was improved in the presence of rGO in PANI Nanorods. Whereas, experimental findings show that the introduction of rGO into PANI improved the photo response from $7 \mu \mathrm{A} \cdot \mathrm{cm}^{-2}$ to $13 \mu \mathrm{A} \cdot \mathrm{cm}^{-2}$. Integration of 3D rGO in PANI results in better photocatalytic performance for the degradation of Congo Red. The enhanced photocatalytic activity with presence of rGO revealed the good potential of PANI-GO nanocomposites for dye degradation. The effective removal of congo red up to $90 \%$ has been observed in acidic medium and is acceptable results compared to the surface area of the substrate. At optimum conditions, also the nature of the antibacterial activities has been investigated by ITO/PANI and ITO/PANI-rGO thin films, and the results have showed exhibited antibacterial activity against the growth of E.coli gram-negative bacteria.

\section{Introduction}

In light of the terrible spread of multiple industries and the widespread use of different toxic elements, the level of pollution in the world increases with days. For this, the protection of the environment by developing global standards increases alongside [1], in order to reach easy and inexpensive solutions to reduce the spread of pollution and try to control it. Many researchers trying to find simple and leading solutions to remove organic compounds from industrial waste that affects the ecosystem and pollutes water [2]. This is what made the global market compete to produce easy-to-manufacture and highly effective materials.

It is now recognized that materials are often the limiting factor in bringing a new technical concept to fruition; thus, conducting and semiconducting polymers are often materials of choice in these demanding applications. Among the most widely employed polymers are polyaniline [3], polypyrrole [4],and polythiophene [5], due to their high conductivity efficiency [6], high stability, unique electrical, optical and electro-optical properties, simple ease of preparation technique ,and also its potential applications.

Moreover, polyaniline (PANI) is one of the most commonly used polymers in many different applications [7-9] and has been widely mentioned in much literature as an effective polymer from semiconductors [10], to eliminate dyes and improve the rate of photocatalytic activity [11, 12]. 
Recently, the photocatalytic degradation technique attracted wide attention for the removal of organic dyes and toxic organic compounds than other methods due to its effectiveness, easy operation, lower cost, non-selective degradation, and high efficiency[13]. Photocatalysis is based on absorbing the light of semiconductor photocatalysts by exciting the electrons from the valence band to the conduction band then create electron-hole pairs[14]. Those electrons and holes degrade the organic dye molecules absorbed on the surface of the photocatalyst by starting a redox reaction with water and oxygen [15].

The high mobility of charge carriers and high absorption efficiency of PANI in visible light indicating good electron donors and electron-hole vectors when excited by visible light [16]. Moreover, to achieve effective results, more efforts are being dedicated to preparing multi-component nanocomposites based on PANI for better functionality efficiency and wider applications rather than being dependent on monocompounds; carbon nanocomposites as graphene [17], graphene oxide [18], and reduced-graphene oxide (rGO) [19] ....etc., are often incorporated due to their high efficiency.

The rGO is one of the best widely used compounds in removing dyes under visible light and photocurrent processes, and has also been widely applied to remove and detect heavy metals it has been mentioned in many literatures [10] thanks to its high active surface area and excellent structural, electronic, mechanical, and optical properties. Ng et al [20] have synthesized in one step a PANI-rGO nanocomposite by electropolymerization process. Thanks to the $\pi-\pi$ interactions of graphene, it can act as a rapid electron transfer channel in the nanocomposite, leading to a remarkable improvement in photoconversion efficiency. Therefore, PANI is attached to the rGO nanosheets to support further photoconversion sites for pollutants ions by nitrogen-containing functional moieties such as amine $(-\mathrm{NH}-)$ and imine $(=\mathrm{N}-)[21,22]$. However, the accumulation of graphene nanosheets with PANI also enhances the mechanical strength of the resulting composites [23].

Interestingly, the bonding between graphene and PANI leads to the strengthens of the quality of conductivity, to solidify more surface recognition sites, and to achieve a high affinity for binding pollutants ions, PANI is combined with rGO [24]. The rGO-based composites have been used for the degradation of various anionic [25] and cationic [26] dyes under visible light.

In addition, also antibacterial properties were reported for PANI and PANI-rGO films [27]. As well-known, graphene and its other derivatives have extraordinary potential for many applications, including biological applications. Several researchers have investigated his antibacterial properties, which are demonstrating their promise in the biomedical and smart food packaging fields [28-30]. Consequently, many studies are being prepared of new nanocomposite thin films by integrated the rGO with a base material, such as PANI to enhancing the antibacterial properties of these nanocomposites and to improve their sterilization capabilities.

Herein, we successfully fabricated efficient electrochemical thins films by combining PANI's electrocatalytic properties and the extended surface sites with high kinetics of charge exchange and high rGO conductivity on the ITO substrate by electrodeposition method. We modified the well-known Hammer method to synthesis a high-quality reduced graphene oxide. Whereas the efficiency of electronic 
transmission of composite nanomaterials was improved by increasing the 3D structure of the rGO groups, which increased their effective surface area. The interaction between nanomaterials, the electrochemical activities of the prepared ITO/PANI and ITO/PANI-rGO composite photocatalyst was tested. Consequently, the behavior of ITO/PANI and ITO/PANI-rGO thins films were studied and their photocatalytic performance was validated to degradation of congo red (CR), analyzed their photocurrent generation, also improved antibacterial applications and its performance.

\section{Experimental Details 2.1. Synthesis of rGO}

First, graphene oxide GO was synthesized chemically via a modified Hummers method [31]. In a typical procedure, sulfuric Acid concentrated $(24 \mathrm{ml})$ was posed in ice bath and added graphite $(1 \mathrm{~g})$ with proper constant stirring. Under vigorous agitation, $3 \mathrm{~g}$ of potassium permanganate $\mathrm{KMnO}_{4}$ was added very slowly to keep the temperature less than $20^{\circ} \mathrm{C}$. Subsequently, the mixture was transferred to a $40^{\circ} \mathrm{C}$ bath with vigorously stirred for $30 \mathrm{~min}$. Then, distilled water $(50 \mathrm{ml})$ was added a slow, with the stirred for 10 $\min$ at $90^{\circ} \mathrm{C}$. The color change to dark-brown which indicates the formation of graphene oxide (GO). A volume of $170 \mathrm{~mL}$ of distilled water was added dropwise followed by slow addition of $\mathrm{H}_{2} \mathrm{O}_{2}(10 \mathrm{~mL}$, $30 \%$ ), after a few seconds, the color of the obtained solution changes to yellow. Finally, the graphene oxide precipitate collected was washed with distilled water and $\mathrm{HCl}$ solution. The resulting solid was airdried and stirred overnight in distilled water, followed by sonication for $25 \mathrm{~min}$. In order to remove the unexfoliated particles, the dispersed GO was centrifuged for 40 min.

After the synthesis of GO, it is transformed into reduced graphene oxide ( $\mathrm{rGO})$. GO (100 mg) was mixed with $100 \mathrm{ml}$ of distilled water giving a yellowish-brown and inhomogeneous dispersion. Complete dispersion on this mixture was carried out using an ultrasonic homogenizer. Then a volume of $1 \mathrm{ml}$ of hydrazine hydrate was added to the solution and the mixture were heated at $105^{\circ} \mathrm{C}$. A black solid precipitate of reduced $O G$ gradually formed. The $\mathrm{rGO}$ is filtered off and washed thoroughly with distilled water and $\mathrm{EtOH}$.

\subsection{Preparation of the thin films}

The electrochemical measurements were made with a potentiostat/galvanostat Autolab PGSTAT 204 at the room temperature. A multi-necked electrochemical cell (three-electrode assembly) were employed for the electrochemical deposition and measurements. The ITO substrates, saturated calomel electrode (SCE), and plate platinum were used as working, reference, and counter electrodes, respectively.

The thin film substrate was prepared following the protocol as shown in Scheme 1. Before starting thin film preparation, the ITO-coated glass substrates $(20 \mathrm{~mm} \times 10 \mathrm{~mm} \times 11 \mathrm{~m})$ were carefully cleaned with acetone and distilled water Din an ultrasonic bath. 
The reduced graphene oxide ( $\mathrm{rGO}$ ) stock solution was prepared by dispersing $20 \mathrm{mg}$ of reduced graphene oxide in $20 \mathrm{ml}$ of distilled water. The electrolyte bath, which was well stirred, was composed of $0.5 \mathrm{M}$ aniline, $1.0 \mathrm{M}$ sulfuric acid and $0.375 \mathrm{mg} / \mathrm{ml} \mathrm{rGO}$. The PANI and PANI-rGO nanocomposites was formed on the working electrodes using cyclic voltammetry (electropolymerization) with a scan rate of $50 \mathrm{mV} \cdot \mathrm{s}^{-1}$. After, the electrodes were carefully rinsed with distilled water.

\subsection{Characterization methods}

The morphology was recorded by Field Emission Scanning Electron Microscopy (JEOL JSM 7600F). Xray photoelectron spectroscopy (XPS) measurements were executed using a Thermo Scientific Kratos Axis Ultra spectrometer with a monochromated AlKa radiation. Optical absorption spectra were carried out at 300-800 nm using UV-Vis spectrophotometer (Cary 60 UV-Vis).

\subsection{Photoelectrochemical Performance}

The photoelectrochemical performance of these electrodes was recorded by illuminating the obtained electrodes from the solution side in an intermittent mode using a Xe lamp. Photocurrent-time responses of ITO/PANI and ITO/PANI-rGO under intermittent light on and off were investigated at increasing applied potentials values in $0.1 \mathrm{M} \mathrm{KCl}$ solution.

\subsection{Photocatalytic activity}

The performance of photocatalytic degradation was investigated using congo red (CR) dye as an organic contaminant. The same-sized ITO/PANI and ITO/PANI-rGO thin film were dipped in a congo red dye. The photocatalytic rate was studied by measuring the color intensity of the CR pollutant. $10 \mathrm{ml}$ of different concentrations of CR solution are placed with ITO/PANI and ITO/PANI-rGO substrates at different irradiation time intervals, and then the Congo Red amount is evaluated using a UV-Vis spectrometer.

\subsection{Antibacterial activities}

The antimicrobial activity of the prepared thin films have been tested against Gram-negative bacteria E.coli ATCC 25922 by using the macrobroth method following the procedure of Time-kill method with some modification [32] by measure the number of surviving microorganisms with time after exposure to the antibacterial agent. This procedure involves preparing freshly suspension, of tested microorganisms from pure spore grown overnight in Nutrient broth at $37^{\circ} \mathrm{C} .1 \mathrm{~mL}$ of each suspension was mixed with 9 $\mathrm{mL}$ of Muller-Hinton agar in sterile test tubes and adjusted to 0.5 Macfarland's standards to obtain about 106 cells $/ \mathrm{mL}$ and then the sterile thin film was put in and incubated at $37^{\circ} \mathrm{C}$ and we also treated $\mathrm{E}$. coli suspension pure PANI and other the E. coli cell aliquots without a simple as a control test. After that, A $100 \mu \mathrm{L}$ aliquot was taken every $12 \mathrm{~h}$ and added to the $500 \mu \mathrm{L}$ of the Muller-Hinton agar in sterile test tubes. Then, the mixture was incubated at $37 \circ \mathrm{C}$ for $24 \mathrm{~h}$. the bacterial colonies at different times were counted to estimate the number of the surviving cells using the colony-forming unit (CFU) method by measuring the optical density of growth by spectrophotometer (Agilent Technologies Cary $60 \mathrm{UV}-\mathrm{Vis}$ ) at $600 \mathrm{~nm}$. 


\section{Results And Discussion}

\subsection{Electropolymerization and deposition studies}

The PANI electropolymerization is based on the redox equations at the working electrode interface. The Basic reactions mechanism can be as follows:

$$
\begin{aligned}
& \mathrm{H}_{2} \mathrm{SO}_{4} \rightarrow 2 \mathrm{H}^{+}+\mathrm{SO}_{4}{ }^{2-} \\
& \mathrm{PANI}+\mathrm{nSO}_{4}{ }^{2-} \rightarrow \mathrm{PANI}^{+2}: \mathrm{SO}_{4}{ }^{2-}+2 \mathrm{ne}^{-} \\
& \mathrm{PANI}^{2+}: \mathrm{SO}_{4}{ }^{2-}+2 \mathrm{ne}^{-} \rightarrow \mathrm{PANI}+\mathrm{nSO}_{4}{ }^{2-}
\end{aligned}
$$

Fig. 1(a) shows the cyclic voltammograms of ITO substrate in an acidic solution containing $0.5 \mathrm{M}$ of aniline. The voltammograms were recorded under scanning potential ranges from $-0.1 \mathrm{~V}$ to $+1.2 \mathrm{~V}$ vs. SCE. The growth of PANI on ITO substrates is shown on the typical with the redox peak attributed located during the potentiodynamic electropolymerization of PANI at around $+0.2 \mathrm{~V}$ and $+0.5 \mathrm{~V}$ for the first cycle. And the following scans in the next cycles showed increasing the peaks corresponding to the oxidations and reductions of the polyaniline films (oxidative peaks ( $\mathrm{a} 1 \mathrm{and} \mathrm{a} 2$ ) coupled with reductive peaks ( $\mathrm{b} 1$ and b2). However, the cyclic voltammograms well agreement with those already reported in the literature on the electro-polymerization of PANI [33]. The pair peaks are observed at 0.3 and $0.9 \mathrm{~V}$ vs. SCE in the third cycle clearly are associated with both polyaniline redox couples from its semiconducting state (leucoemeraldine) and to its conductive state (emeraldine), and the emeraldine to its full oxidation form (pernigraniline) [34].

Fig. 1 (b) shows the voltammogram of ITO/PANI-rGO thin films recorded between -0.56 and $1.2 \mathrm{~V}$ vs. SCE to favor the nanocomposites growth. During the electropolymerization process of PANI film, rGO diffused slowly to the PANI sites simultaneously where redox reactions take place. The good solubility and stability of the rGO suspension in the electrolyte allows a better insertion of rGO in the PANI and to obtain more homogeneous thin films.

The rGO is formed in situ with the PANI on the ITO substrate after ion exchange subjected to ten 10 scans in the potential range, shows two pairs of redox peaks increase continuously with each cycle due to the transition between quinone/hydroquinone groups in rGO, which is typical for carbon [35]. By these functional groups of rGO, aniline monomers can be attached to rGO nanosheets (Insert Fig. 1(b) has been showing the formation groups of rGO in the 3D PANI network by FESEM characterization). Moreover, the rGO nanosheets have good conductivity, which helped dramatically to increase the current of the voltammogram cycles as shown in Fig. 1(b). The Cycles voltammograms profile Fig. 1(c) shows the oxidation peak of PANI-rGO located at $0.3 \mathrm{~V}$ and $0.5 \mathrm{~V}$ vs. SCE and the reduction peak at $0.05 \mathrm{~V}$ and $0.3 \mathrm{vs}$. SCE for the first and last cycles. The large transition between the first and the last cycle demonstrates the increased electroactivity of the growth of the PANI-rGO film on the surface of ITO 
compared to the voltammogram of Fig. 1(a) thus confirming the better conductivity of the PANI/rGO film, and allowed improve the electrical performance of the film. Although the high conductivity of PANI, the rGO sheets improves a high contact interface between the PANI and the electrolyte [36] and thus explained the difference in the voltammogram cycles to the formation of PANI-rGO film with high specific surface area.

\subsection{Surface morphological analysis}

FESEM images was used to characterize the morphologies of the elaborated thin films, as indicated in Fig.2. The Fig. 2(a,b) shows the appearance of the rGO surface and confirms the deposition of rGO layers on ITO. As can be observed in Fig. 2(b), wrinkle-like structure, crumpled, thin and agglomerated sheets closely associated with each other. The wrinkled structure observed of rGO sheets is due to the removal of intercalated functional groups in graphitic oxide during exfoliation. An average thickness of several nanometers significantly increased the thin film effective surface area. Thus this result confirms that hydrazine hydrates have chemically reduced GO to form rGO [37].

While Fig. 2 (c,d) shows the surface morphology of the ITO/PANI thin film, which constitutes an interconnected network of PANI nanorods (NRs) represent a well-arranged and orderly uniform polymer chain structure. which makes this network useful for electron transport at the electrolyte-thin film interface [38-40]. Fig. 2(d) shows an enlarged view of ITO/PANI nanorods, which clearly shows the diameter of the PANI (around $110 \mathrm{~nm}$ ) and the length of uniform size about $1 \mathrm{~mm}$. The acquisition of a more regular and uniform microstructure offers more active sites for the fixing of pollutants.

Figure 2(e) and Fig. 2(f) show the surface morphology of the ITO/PANI-rGO and enlarged view of that thin film, respectively. It is noticeable in Fig. 2(e) that the layers of rGO that have been covered by PANI NRs are formed well-arranged with evenly dispersed. In Fig. 2(f), with an enlarged view, we could observe the nanorods of PANI that largely cover the layers of $\mathrm{rGO}$ and are embedded with them. We can say that it has formed bonds interaction between rGO and PANI NRs, this is what facilitates the electron transfer process. The point of the merging process is to form a fertile platform for the transfer of electrons and accelerate the electrical response.

\subsection{XPS and UV-Vis analysis}

Fig.3. Shows the XPS spectra of ITO-PANI and ITO/PANI-rGO electrodes. As is visible from Fig. 3(a), and through the presented materials, the peaks centered at $290 \mathrm{eV}$ (C1s), $400 \mathrm{eV}$ (N1s), and $540 \mathrm{eV}$ (01s) are all visible a wide range in the spectrum. The presence of N1s confirms the presence of polyaniline in all synthesized nanocomposites. Fig. 3(b, c) displays the deconvoluted C1s peak of PANI and PANI-rGO, revealing the presence of three different types of carbon functional groups: nonoxygenated carbon , nitrogenated carbon and oxygenated carbon $[41,42]$.

Fig. 4 Represents the optical absorption spectra of ITO/rGO, ITO/PANI, and ITO/ PANI-rGO films. The peak in the spectra of rGO at 340 due to the $\pi-\pi^{\star}$ absorption band demonstrates the elimination of oxygen 
functions in the outer portion of the $\mathrm{GO}$ and the electron conjugations of $\mathrm{C}-\mathrm{C}$ in the basal levels of the recovered GO rendering it as $\mathrm{rGO}[43,44]$. PANI exhibits well-defined three strong absorption peaks at approximately 371,427 , and $773 \mathrm{~nm}$ correspond to the formation of polyaniline. The peaks observed at 317 and $427 \mathrm{~nm}$ are attributed to the transition of electrons from the HOMO to the LUMO. These peaks correspond to the $\pi-\pi^{\star}$ electronic transitions of the bipolar ion, benzenoid rings and polar ion excitations of the quinoid rings. The peak at $773 \mathrm{~nm}$ is credited to the $n-\pi^{\star}$ electronic transitions between benzenoid and quinoid units [45-47]. In the UV-Vis spectra of the ITO/PANI-rGO electrode, two kinds of characteristic peaks were observed at $371 \mathrm{~nm}$ and $410 \mathrm{~nm}$ which are attributed to the PANI and rGO respectively.

\subsection{Photoelectrochemical performance}

The photoelectrochemical performance of ITO/PANI and ITO/PANI-rGO nanocomposites is estimated according to the ability to absorb light under intermittent light on and off. It is noticed in Fig.5(a), that the current density under dark conditions is straight and near to zero, whereas, under illumination, it is observed that there is a significant increase in photocurrent with a rectangular vertically response. Rapid and uniform photocurrent responses to all switch-on and switch-off cases were observed across all electrodes. The positive values of photocurrents are a characteristic of the n-type semiconductor $[48,49]$, this is explained by the presence of charge carriers that transfer from HOMO to LUMO under light irradiation [50,51]. As the lighting continues, the generation of photo-excited electrons annihilates the internal electric field, as electrons and holes accumulate and lead to recombination of the charge [52]. It was noted that the photocurrent intensity of the PANI-rGO electrodes was significantly improved compared to the pure PANI electrodes.

Fig.5(b) shows the photocurrent variation as a function of the applied potential for the synthesized ITO/PANI-rGO. A range of different potentials has been used to study thin film photocurrent performance. It has been shown that $\mathrm{rGO}$ with high electron conductivity could aid electron transfer and suppressing the electron-hole combination [53], reaching a much higher photocurrent and faster photo response. According to Henni et al. [37] the rGO may have led to the broadening of the band gap of the PANI-rGO sample by increasing the carrier intensity in the valence band. However, it is observed that the photocurrent intensity of the electrodes increases reasonably with the increase in the potential range.

\subsection{Photocatalytic activity}

The prepared ITO/PANI and ITO/PANI-rGO thin films photocatalysts were investigated by following the degradation of $\mathrm{CR}$ pollutants under light irradiation with studies influence of $\mathrm{pH}$ and the temperature into the degradation of dyes organic (Fig. 6). First the maximum absorbance of the dye, in the absence of any catalysts at $\mathrm{T}=30^{\circ} \mathrm{C}$ and $\mathrm{pH}=6.05$, was measured scanned in the range of $300-800 \mathrm{~nm}$ and it was observed at a wavelength of $498 \mathrm{~nm}$ ascribed to Congo Red dye. The resulting spectrum is shown in Fig. 6(a). The change of absorbance was used to evaluate the photo-degradation efficiency. For 10 min in the dark, the adsorption process is achieved for adsorption-desorption equilibrium before exposing the CR to irradiation. 
It is observed in Fig. 6(b) that the degradation rate of CR dye increased well using ITO/PANI-rGO compared to ITO/PANI electrodes. Rapid degradation has been observed for ITO/PANI-rGO electrodes. After $60 \mathrm{~min}$, the degradation of the $\mathrm{CR}$ for reached close to 70\%. As for the ITO/PANI electrodes, the degradation of $\mathrm{CR}$ reached $40 \%$. Regarding the ITO/PANI-rGO curves, it is noticeable that the degradation rate of CR suspension increased well compared to the curves of ITO / PANI. The presence of rGO in the polyaniline structure has enhanced the photocatalytic activity, this is explained by the synergistic effect between rGO and PANI. The same effect was observed in our previous study when the rGO has been incorporated in ZnO thin layers [37] and El-Sharkaway E. A, et al [19] reported that PANI/rGO composites show a higher adsorption capacity of MB dye compared with the PANI adsorbents. Therefore, the improved substrate performances can be assigned to the interesting electroactive properties of PANI and the excellent electrical conductivity of rGO $[54,55]$.

The $\mathrm{pH}$ factor also considered a very important factor and helps speed up and changed the photocatalytic process. The photocatalytic degradation activity of CR by ITO/PANI and ITO/PANI-rGO at different $\mathrm{pH}$ is shown in Fig. 6(c). It is observed that the $\mathrm{CR}$ showed high degradation efficiency in a short irradiation time at an acidic medium (reached about $90 \%$ for both ITO/PANI and ITO/PANI-rGO). This is due to the PANI behavior that works well in an acidic medium compared to the basic medium. While in the medium with $\mathrm{pH}=7$, the degradation rate was close to $60 \%$ for ITO/PANI and $75 \%$ for ITO/PANI-rGO.

Fig. 6 (d) shows the effect of temperature on the photocatalytic process. It observed that at $T=20^{\circ} \mathrm{C}$ the degradation rate was $58 \%$ and $66 \%$ for ITO/PANI and ITO/PANI-rGO respectively, and while the temperature increased to $\left(T=40^{\circ} \mathrm{C}\right)$, the rate of degradation increased and reached $80 \%$ for ITO/PANI-rGO while maintaining the same conditions. While it reached more than $80 \%$ for the ITO/PANI-rGO while continuing to rise in temperature $\left(T=60^{\circ} \mathrm{C}\right)$. The effect of temperature plays a very important role in photocatalytic processes as a catalyst and accelerator for the degradation of dyes. The photocatalytic mechanism of CR degradation is presented in Fig. 6(e).

The point of zero charge $\mathrm{pH}_{\mathrm{pzc}}$ is very important in studying the adsorption mechanism process as well as determining the effect of $\mathrm{pH}$ on adsorption. When the $\mathrm{pH}$ is higher than $\mathrm{pH}_{\mathrm{pzc}}$, the catalyst surface is negatively charged and attracts cations, while it is positively charged and repels cations at $\mathrm{pH}$ lower than $\mathrm{pH}_{\mathrm{pzc}}$ [56][57]. The $\mathrm{pH}_{\mathrm{pzc}}$ of ITO/PANI and ITO/PANI-rGO was found to be 2.76 and 3.09 respectively as shown in Fig. 7(a,b). Since the adsorbent surface was positively charged according to the results, ITO/PANI and ITO/PANI-rGO preferred to remove the color dye at $\mathrm{pH}>\mathrm{pH}_{\mathrm{pzc}}$.

The adsorption kinetics of ITO/PANI and ITO/PANI-rGO was examined for CR by plotting the Ct/Co values on the function of irradiation time (Fig. 6(c)). Lower values for ITO/PANI-rGO electrodes of Ct/Co have been obtained which confirmed that the affinity of the dye pollutant towards the ITO/PANI-rGO thin film surface with enhanced photocatalytic degradation of $C R$ at the surface. The plot of $\ln \left(C_{0} / C_{t}\right)$ against $t$ (Fig. 6(d)) display a straight line, indicating the good coincidence with the pseudo first-order equation and suggesting that the nanocomposite (ITO/PANI and ITO/PANI-rGO) owns high catalytic activity. On the other hand, the correlation coefficient of the pseudo-first-order model nearly $\mathrm{R}^{2}=0.99$ for all electrodes. 


\subsection{Antibacterial activity}

Antibacterial activities of the ITO/PANI and ITO/PANI-rGO thin films were studied against one Gramnegative bacterium E. coli ATCC 25922 by the time-kill method. The results of the antimicrobial assay at the following contact times are presented as CFU of surviving cells ant rate of reduction compared with the negative test without simple as shown in Fig. $8(a, b)$. The results showed clearly that, all thin films exhibited antibacterial activity against the growth of $E$. coli gram-negative bacterial. In contrast, treatment with PANI thin film showed a statistically significant reduction in the growth of cells compared with untreated cells as reported by recent studies [58-61]. Furthermore, a noticeable difference was observed between the effect of each nanocomposite, Antibacterial efficiency of ITO/PANI/rGO was higher than ITO/PANI, and that seems similar to the resultants reported by Lin Shi [62]. Moreover, the ITO/PANI-rGO demonstrated the higher bactericidal activity with a $50 \%$ of reduction in numbers of $E$. coli after $60 \mathrm{~h}$ of exposure compared with ITO/PANI (33\%), suggesting a synergistic effect of rGO and PANI. Can say that the results are acceptable compare with the area of the substrate.

\section{Conclusion}

In this study, ITO/ PANI and ITO/PANI-rGO thin films were successfully prepared and by electropolymerization of aniline in the presence of reduced graphene oxide. The chemical states and morphological analysis of synthesized PANI-rGO nanocomposite have been studied comprehensively. The elaborated nanocomposites were tested to be effective adsorbers for congo red dye and to improve photocurrent generation. The synergistic effect between PANI and rGO in the nanocomposite improved light catalytic activity compared with PANI alone due to the superior separation efficiency of the electronhole pairs. In the acid and basic medium, the PANI-rGO thin film has exhibited an effective behavior in removing the dye. In comparison with the PANI nanorods, the rGO-PANI composite demonstrated an interesting improvement in generated photocurrent. The PANI-rGO composite is a promising material for the degradation processes of dye pollutants. The coupling of the polyaniline with the reduced graphene oxide removed $77 \%$ of the dye after $120 \mathrm{~min}$, and $90 \%$ in the acidic medium. The elaborated thin film exhibited a good antibacterial activity against the growth of $E$. coli bacteria.

\section{References}

1. K.C. Lai, K.H. Yeap, S.K. Lim, P.C. Teh, H. Nisar, An Investigation on Food Waste Recovery: A Preliminary Step of Waste-to-Energy (WtE) Development, Energy Procedia. 138 (2017) 169-174

2. X. Li, J. Xie, C. Jiang, J. Yu, P. Zhang, Review on design and evaluation of environmental photocatalysts. Front. Environ. Sci. Eng. 12, 1-32 (2018)

3. N. Harfouche, B. Nessark, F.X. Perrin, Electrochemical and surface characterization of composite material: Polyaniline/LiMn2O4. J. Electroanal. Chem. 756, 179-185 (2015). doi:10.1016/j.jelechem.2015.08.031 
4. L. Jia, X. Wei, L. Lv, X. Zhang, X. Duan, Y. Xu, K. Liu, J. Wang, Electrodeposition of hydroxyapatite on nickel foam and further modification with conductive polyaniline for non-enzymatic glucose sensing. Electrochim. Acta 280, 315-322 (2018). doi:10.1016/j.electacta.2018.05.130

5. J. Zhang, S. Cao, S. Xu, H. Yang, L. Yang, Y. Song, L. Jiang, Y. Dan, Study on stability of poly (3hexylthiophene)/titanium dioxide composites as a visible light photocatalyst. Appl. Surf. Sci. 349, 650-656 (2015)

6. H.M. Mousa, J.R. Aggas, A. Guiseppi-Elie, Electropolymerization of aniline and (N-phenyl-ophenylenediamine) for glucose biosensor application. Mater. Lett. 238, 267-270 (2019). doi:10.1016/j.matlet.2018.12.012

7. A. Umar, A.A. Ibrahim, H. Algadi, H. Albargi, M.A. Alsairi, Y. Wang, S. Akbar, Enhanced NO2 gas sensor device based on supramolecularly assembled polyaniline/silver oxide/graphene oxide composites. Ceram. Int. (2021). doi:10.1016/j.ceramint.2021.05.296

8. V. Sharma, V. Maivizhikannan, V.N. Rao, S. Kumar, A. Kumar, A. Kumar, M.V. Shankar, V. Krishnan, Sea urchin shaped $\mathrm{ZnO}$ coupled with MoS2 and polyaniline as highly efficient photocatalysts for organic pollutant decomposition and hydrogen evolution. Ceram. Int. 47, 10301-10313 (2021).

doi:10.1016/j.ceramint.2020.09.199

9. Y.-S. Fang, P. He, Y.-Z. Cai, W.-Q. Cao, M.-S. Cao, Bifunctional Ti3C2Tx-CNT/PANI composite with excellent electromagnetic shielding and supercapacitive performance. Ceram. Int. (2021). doi:10.1016/j.ceramint.2021.05.277

10. M. Mitra, S.T. Ahamed, A. Ghosh, A. Mondal, K. Kargupta, S. Ganguly, D. Banerjee, Polyaniline/Reduced Graphene Oxide Composite-Enhanced Visible-Light-Driven Photocatalytic Activity for the Degradation of Organic Dyes. ACS Omega 4, 1623-1635 (2019). doi:10.1021/acsomega.8b02941

11. M. Hassan, K.R. Reddy, E. Haque, S.N. Faisal, S. Ghasemi, A.I. Minett, V.G. Gomes, Hierarchical assembly of graphene/polyaniline nanostructures to synthesize free-standing supercapacitor electrode. Compos. Sci. Technol. 98, 1-8 (2014)

12. S. Bai, Y. Tian, M. Cui, J. Sun, Y. Tian, R. Luo, A. Chen, D. Li, Polyaniline@ SnO2 heterojunction loading on flexible PET thin film for detection of NH3 at room temperature. Sensors Actuators B Chem. 226, 540-547 (2016)

13. N.M. Aboamera, A. Mohamed, A. Salama, T.A. Osman, A. Khattab, An effective removal of organic dyes using surface functionalized cellulose acetate/graphene oxide composite nanofibers. Cellulose. 25, 4155-4166 (2018). doi:10.1007/s10570-018-1870-8

14. S.A. Karim, A. Mohamed, M.M. Abdel-Mottaleb, T.A. Osman, A. Khattab, Mechanical Properties and the Characterization of Polyacrylonitrile/Carbon Nanotube Composite Nanofiber., Arab. J. Sci. Eng. (Springer Sci. Bus. Media BV). 43 (2018)

15. A. Mohamed, W.S. Nasser, T.A. Osman, M.S. Toprak, M. Muhammed, A. Uheida, Removal of chromium $(\mathrm{VI})$ from aqueous solutions using surface modified composite nanofibers. J. Colloid Interface Sci. 505, 682-691 (2017) 
16. K.-C. Huang, J.-H. Huang, C.-H. Wu, C.-Y. Liu, H.-W. Chen, C.-W. Chu, C.-L. Lin, K.-C. Ho, Nanographite/polyaniline composite films as the counter electrodes for dye-sensitized solar cells. J. Mater. Chem. 21, 10384-10389 (2011)

17. D.A. Pethsangave, R.V. Khose, P.H. Wadekar, D.K. Kulal, S. Some, One-Pot Synthetic Approach for Magnetically Separable Graphene Nanocomposite for Dye Degradation, ChemistrySelect. 5 (2020) 1516-1525

18. C. Lv, J. Zhang, G. Li, H. Xi, M. Ge, T. Goto, Facile fabrication of self-assembled lamellar PANI-GOFe304 hybrid nanocomposites with enhanced adsorption capacities and easy recyclicity towards ionic dyes, Colloids Surfaces A Physicochem. Eng. Asp. 585, 124147 (2020)

19. E.A. El-Sharkaway, R.M. Kamel, I.M. El-Sherbiny, S.S. Gharib, Removal of methylene blue from aqueous solutions using polyaniline/graphene oxide or polyaniline/reduced graphene oxide composites, Environ. Technol. (2019)

20. Y.H. Ng, A. Iwase, N.J. Bell, A. Kudo, R. Amal, Semiconductor/reduced graphene oxide nanocomposites derived from photocatalytic reactions. Catal. Today 164, 353-357 (2011). doi:10.1016/j.cattod.2010.10.090

21. X. Wang, J. Zhang, K. Zhang, W. Zou, S. Chen, Facile fabrication of reduced graphene oxide/Cul/PANI nanocomposites with enhanced visible-light photocatalytic activity. RSC Adv. 6, 44851-44858 (2016)

22. M. Akhtar, A. Tahir, S. Zulfiqar, F. Hanif, M.F. Warsi, P.O. Agboola, I. Shakir, Ternary hybrid of polyaniline-alanine-reduced graphene oxide for electrochemical sensing of heavy metal ions. Synth. Met. 265, $116410(2020)$

23. M. Ates, M. El-Kady, R.B. Kaner, Three-dimensional design and fabrication of reduced graphene oxide/polyaniline composite hydrogel electrodes for high performance electrochemical supercapacitors. Nanotechnology. 29, 175402 (2018)

24. F. Hanif, A. Tahir, M. Akhtar, M. Waseem, S. Haider, M.F.A. Aboud, I. Shakir, M. Imran, M.F. Warsi, Ultraselective detection of $\mathrm{Cd} 2+$ and $\mathrm{Pb} 2+$ using glycine functionalized reduced graphene oxide/polyaniline nanocomposite electrode. Synth. Met. 257, 116185 (2019)

25. W. Yin, S. Hao, H. Cao, Solvothermal synthesis of magnetic CoFe 2 O 4/rGO nanocomposites for highly efficient dye removal in wastewater. RSC Adv. 7, 4062-4069 (2017)

26. S.K. Maji, A. Jana, Two-dimensional nanohybrid (RGS@AuNPs) as an effective catalyst for the reduction of 4-nitrophenol and photo-degradation of methylene blue dye. New J. Chem. 41, 33263332 (2017)

27. S. Ghobadi, S. Mehraeen, R. Bakhtiari, B. Shamloo, V. Sadhu, M. Papila, F. Cebeci, S.A. Gürsel, PVA/PANI/rGO ternary electrospun mats as metal-free anti-bacterial substrates. RSC Adv. 6, 9243492442 (2016)

28. Z. Jia, W. Xu, Synthesis and antibacterial activities of quaternary ammonium salt of chitosan. Carbohydr. Res. 333, 1-6 (2001) 
29. J. Zhao, B. Deng, M. Lv, J. Li, Y. Zhang, H. Jiang, C. Peng, J. Li, J. Shi, Q. Huang, Graphene oxidebased antibacterial cotton fabrics. Adv. Healthc. Mater. 2, 1259-1266 (2013)

30. J. He, X. Zhu, Z. Qi, C. Wang, X. Mao, C. Zhu, Z. He, M. Li, Z. Tang, Killing dental pathogens using antibacterial graphene oxide. ACS Appl. Mater. Interfaces. 7, 5605-5611 (2015)

31. W.S. Hummers Jr., R.E. Offeman, Preparation of graphitic oxide. J. Am. Chem. Soc. 80, 1339 (1958)

32. A.P. MacGowan, M. Wootton, A.J. Hedges, K.E. Bowker, H.A. Holt, D.S. Reeves, A new time-kill method of assessing the relative efficacy of antimicrobial agents alone and in combination developed using a representative $\beta$-lactam, aminoglycoside and fluoroquinolone. J. Antimicrob. Chemother. 38, 193203 (1996)

33. C.C. Buron, B. Lakard, A.F. Monnin, V. Moutarlier, S. Lakard, Elaboration and characterization of polyaniline films electrodeposited on tin oxides. Synth. Met. 161, 2162-2169 (2011). doi:10.1016/j.synthmet.2011.08.021

34. A. Sayah, F. Habelhames, A. Bahloul, B. Nessark, Y. Bonnassieux, D. Tendelier, M.El Jouad, Electrochemical synthesis of polyaniline-exfoliated graphene composite films and their capacitance properties. J. Electroanal. Chem. 818, \#pagerange\# (2018). doi:10.1016/j.jelechem.2018.04.016

35. J.M. Sieben, A. Ansón-Casaos, F. Montilla, M.T. Martínez, E. Morallon, Electrochemical behaviour of different redox probes on single wall carbon nanotube buckypaper-modified electrodes. Electrochim. Acta 135, 404-411 (2014)

36. D.-W. Wang, F. Li, J. Zhao, W. Ren, Z.-G. Chen, J. Tan, Z.-S. Wu, I. Gentle, G.Q. Lu, H.-M. Cheng, Fabrication of graphene/polyaniline composite paper via in situ anodic electropolymerization for high-performance flexible electrode. ACS Nano 3, 1745-1752 (2009)

37. A. Henni, N. Harfouche, A. Karar, D. Zerrouki, F.X. Perrin, F. Rosei, Synthesis of graphene-ZnO nanocomposites by a one-step electrochemical deposition for efficient photocatalytic degradation of organic pollutant. Solid State Sci. 98, 106039 (2019). doi:10.1016/j.solidstatesciences.2019.106039

38. L. Shao, X. Wang, B. Yang, Q. Wang, Q. Tian, Z. Ji, J. Zhang, A highly sensitive ascorbic acid sensor based on hierarchical polyaniline coated halloysite nanotubes prepared by electrophoretic deposition. Electrochim. Acta 255, 286-297 (2017)

39. V.T. Thu, B.Q. Tien, D.T. Ngoc Nga, L.C. Thanh, L.H. Sinh, T.C. Le, T.D. Lam, Reduced graphene oxidepolyaniline film as enhanced sensing interface for the detection of loop-mediated-isothermalamplification products by open circuit potential measurement. RSC Adv. 8, 25361-25367 (2018). doi:10.1039/c8ra04050h

40. M. Xu, Y. Song, Y. Ye, C. Gong, Y. Shen, L. Wang, L. Wang, A novel flexible electrochemical glucose sensor based on gold nanoparticles/polyaniline arrays/carbon cloth electrode. Sensors Actuators B Chem. 252, 1187-1193 (2017)

41. Y. Fan, J.-H. Liu, C.-P. Yang, M. Yu, P. Liu, Graphene-polyaniline composite film modified electrode for voltammetric determination of 4-aminophenol. Sensors Actuators B Chem. 157, 669-674 (2011)

42. C. Lamiel, D. Kharismadewi, J.-J. Shim, Covalently bonded reduced graphene oxide/polyaniline composite for electrochemical sensors and capacitors. J. Electroanal. Chem. 758, 148-155 (2015) 
43. R. Krishna, E. Titus, O. Okhay, J.C. Gil, J. Ventura, E.V. Ramana, J.J.A. Gracio, Rapid electrochemical synthesis of hydrogenated graphene oxide using Ni nanoparticles. Int. J. Electrochem. Sci. 9, 40544069 (2014)

44. Z. Çiplak, N. Yildiz, A. Çalimli, Investigation of graphene/Ag nanocomposites synthesis parameters for two different synthesis methods, Fullerenes, Nanotub. Carbon Nanostructures. 23, 361-370 (2015)

45. M. Alam, N.M. Alandis, A.A. Ansari, M.R. Shaik, Optical and electrical studies of polyaniline/ZnO nanocomposite, J. Nanomater. 2013 (2013)

46. H.S. Abdulla, A.I. Abbo, Optical and electrical properties of thin films of polyaniline and polypyrrole. Int. J. Electrochem. Sci. 7, 10666-10678 (2012)

47. S. Goswami, S. Nandy, T.R. Calmeiro, R. Igreja, R. Martins, E. Fortunato, Stress induced mechanoelectrical writing-reading of polymer film powered by contact electrification mechanism. Sci. Rep. 6, 19514 (2016)

48. A. Henni, A. Merrouche, L. Telli, A. Azizi, R. Nechache, Effect of potential on the early stages of nucleation and properties of the electrochemically synthesized ZnO nanorods. Mater. Sci. Semicond. Process. 31, 380-385 (2015). doi:10.1016/j.mssp.2014.12.011

49. A. Mahroug, B. Mari, M. Mollar, I. Boudjadar, L. Guerbous, A. Henni, N. Selmi, Studies on structural, surface morphological, optical, luminescence and Uv photodetection properties of sol-gel Mg-doped ZnO thin films, Surf. Rev. Lett. (2018) 1850167. doi:10.1142/S0218625X18501676

50. Y. Bouznit, A. Henni, Characterization of $\mathrm{Sb}$ doped $\mathrm{SnO} 2$ films prepared by spray technique and their application to photocurrent generation. Mater. Chem. Phys. 233, 242-248 (2019). doi:10.1016/j.matchemphys.2019.05.072

51. Y. Bouznit, A. Henni, Enhanced photoelectrochemical performance of Al-doped ZnO thin films prepared by co-spray technique. Mater. Sci. Semicond. Process. 118, 105208 (2020). doi:10.1016/j.mssp.2020.105208

52. W. Zhang, H. Guo, H. Sun, R. Zeng, Constructing ternary polyaniline-graphene-TiO2 hybrids with enhanced photoelectrochemical performance in photo-generated cathodic protection. Appl. Surf. Sci. 410, 547-556 (2017)

53. W. Ma, D. Han, S. Gan, N. Zhang, S. Liu, T. Wu, Q. Zhang, X. Dong, L. Niu, Rapid and specific sensing of gallic acid with a photoelectrochemical platform based on polyaniline-reduced graphene oxideTiO2. Chem. Commun. 49, 7842 (2013). doi:10.1039/c3cc43540g

54. T. Guo, L. Wang, D.G. Evans, W. Yang, Synthesis and Photocatalytic Properties of a Polyanilineintercalated layered protonic titanate nanocomposite with ap $-\mathrm{n}$ heterojunction structure. J. Phys. Chem. C 114, 4765-4772 (2010)

55. O. Akhavan, The effect of heat treatment on formation of graphene thin films from graphene oxide nanosheets. Carbon N. Y. 48, 509-519 (2010)

56. H.A. Ghaly, A.S. El-Kalliny, T.A. Gad-Allah, N.E.A. Abd El-Sattar, E.R. Souaya, Stable plasmonic $\mathrm{Ag} / \mathrm{AgCl}$-polyaniline photoactive composite for degradation of organic contaminants under solar 
light. RSC Adv. 7, 12726-12736 (2017)

57. O. Bechambi, S. Sayadi, W. Najjar, Photocatalytic degradation of bisphenol A in the presence of Cdoped $\mathrm{ZnO}$ : effect of operational parameters and photodegradation mechanism. J. Ind. Eng. Chem. 32, 201-210 (2015)

58. N. Shi, X. Guo, H. Jing, J. Gong, C. Sun, K. Yang, Antibacterial effect of the conducting polyaniline. J. Mater. Sci. Technol. 22, 289-290 (2006)

59. M.R. Gizdavic-Nikolaidis, J.R. Bennett, S. Swift, A.J. Easteal, M. Ambrose, Broad spectrum antimicrobial activity of functionalized polyanilines. Acta Biomater 7, 4204-4209 (2011)

60. E.F. Palermo, K. Kuroda, Structural determinants of antimicrobial activity in polymers which mimic host defense peptides. Appl. Microbiol. Biotechnol. 87, 1605-1615 (2010)

61. M.R. Gizdavic-Nikolaidis, J. Bennett, Z. Zujovic, S. Swift, G.A. Bowmaker, Characterization and antimicrobial efficacy of acetone extracted aniline oligomers. Synth. Met. 162, 1114-1119 (2012)

62. L. Shi, J. Chen, L. Teng, L. Wang, G. Zhu, S. Liu, Z. Luo, X. Shi, Y. Wang, L. Ren, The antibacterial applications of graphene and its derivatives. Small. 12, 4165-4184 (2016)

\section{Figures}



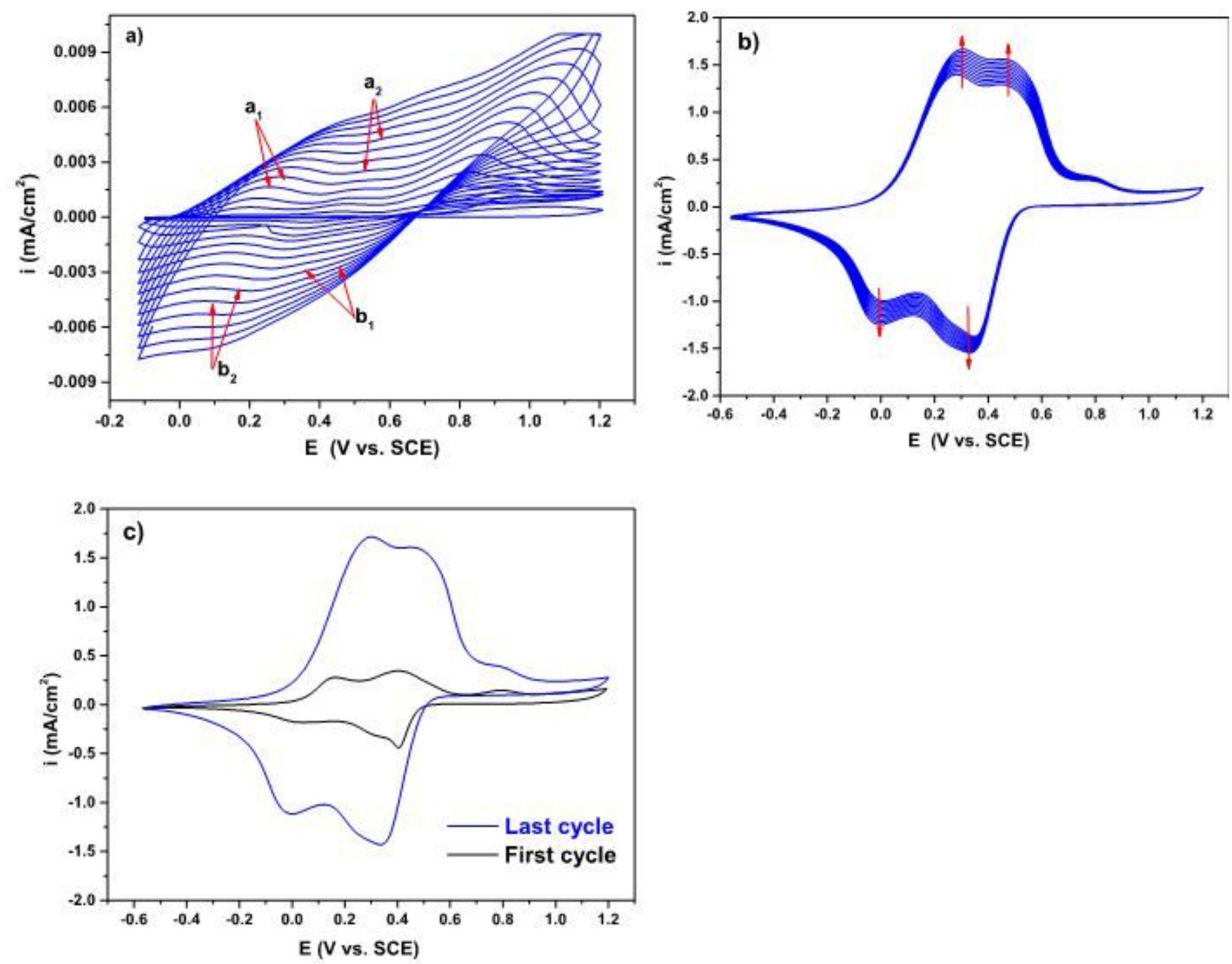

Figure 1

Cyclic voltammograms of (a) PANI and (b) PANI coupled with rGO on ITO substrate. (c) The first and the last cycles of the PANI/rGO thin film. 

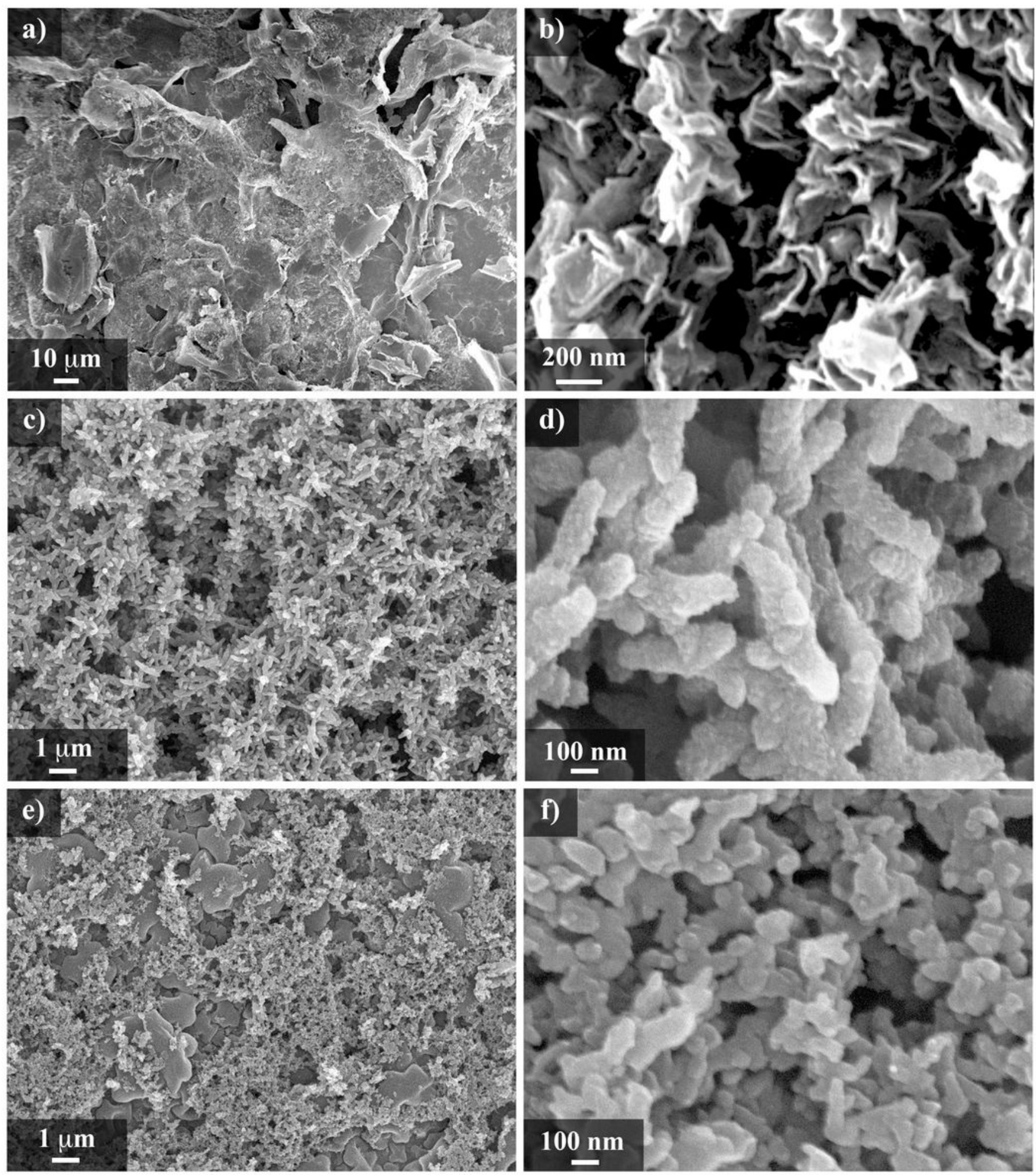

Figure 2

FESEM images of the thin films: $(a, b)$ ITO/rGO layers, $(b, c)$ ITO/PANI nanorods and $(d, e)$ ITO/PANI-rGO. 

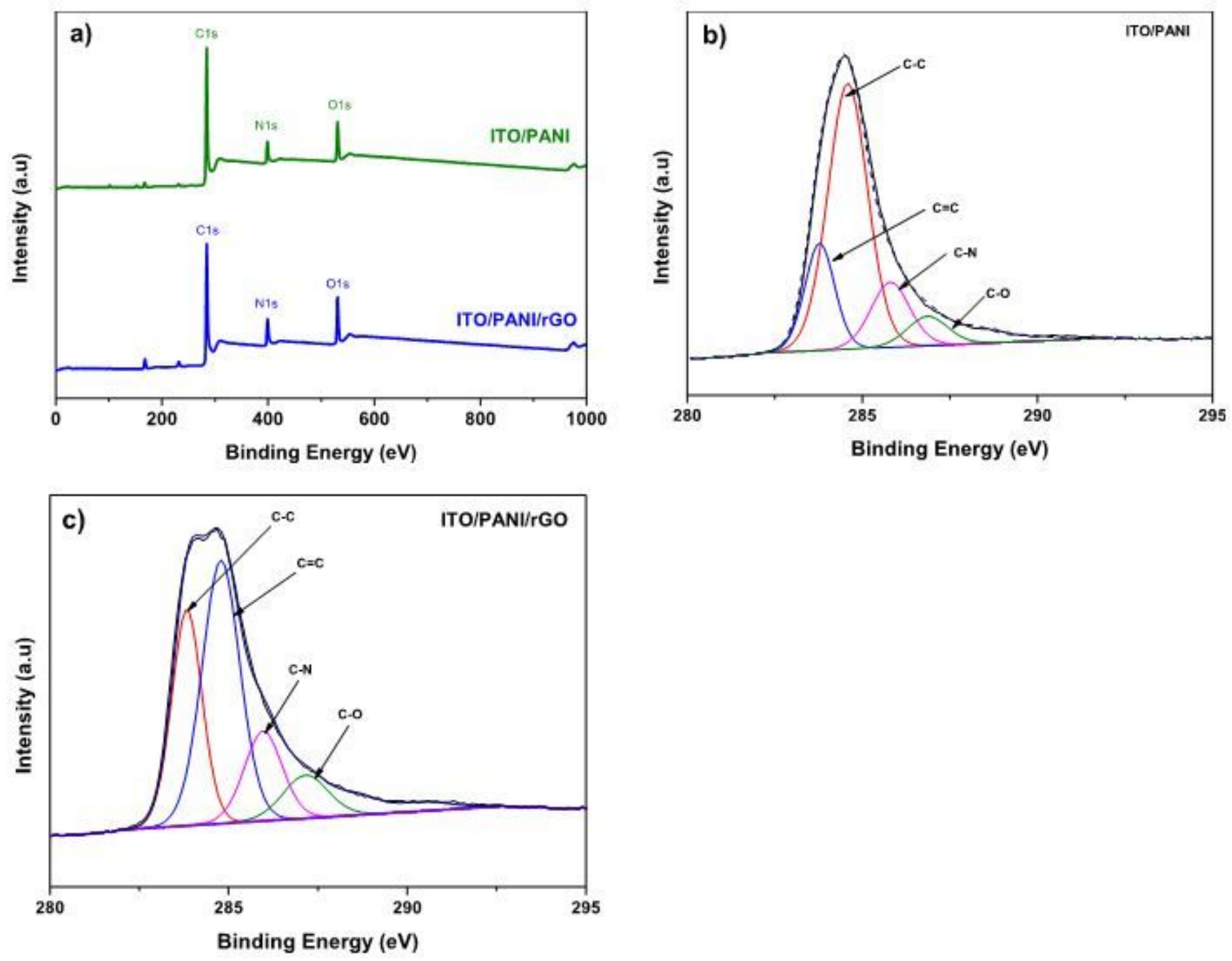

Figure 3

(a) XPS spectra of ITO/PANI and ITO/PANI/rGO. (b) C1s XPS spectra of ITO/PANI and (c) C1s XPS spectra of ITO/PANI-rGO. 


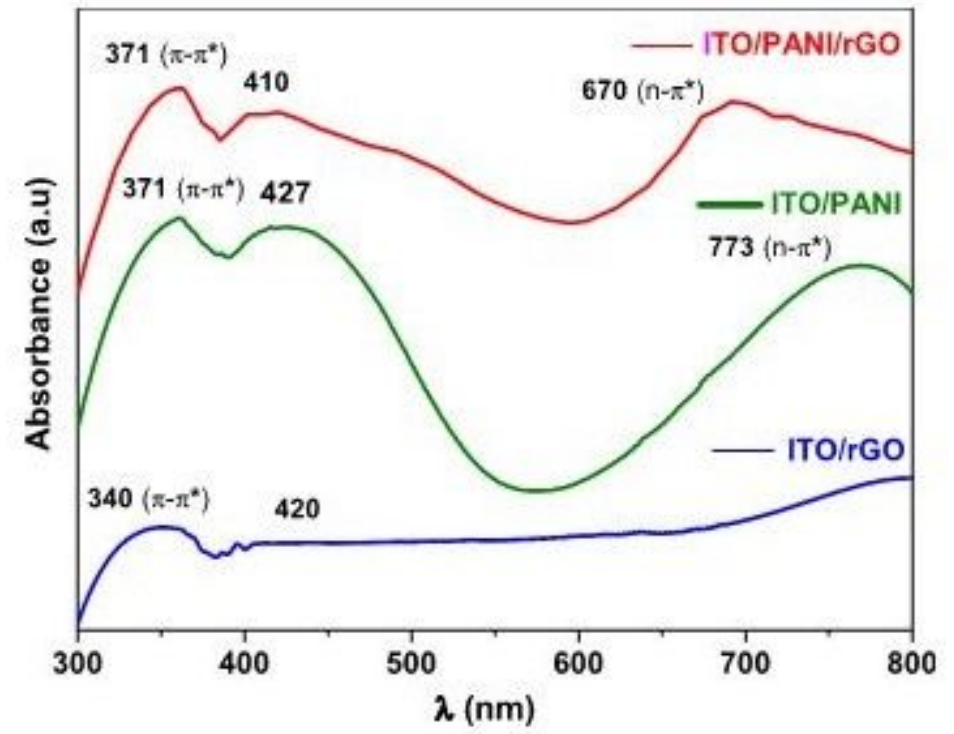

Figure 4

UV-Visible spectra of ITO/rGO, ITO/PANI, and ITO/PAN-rGO. 

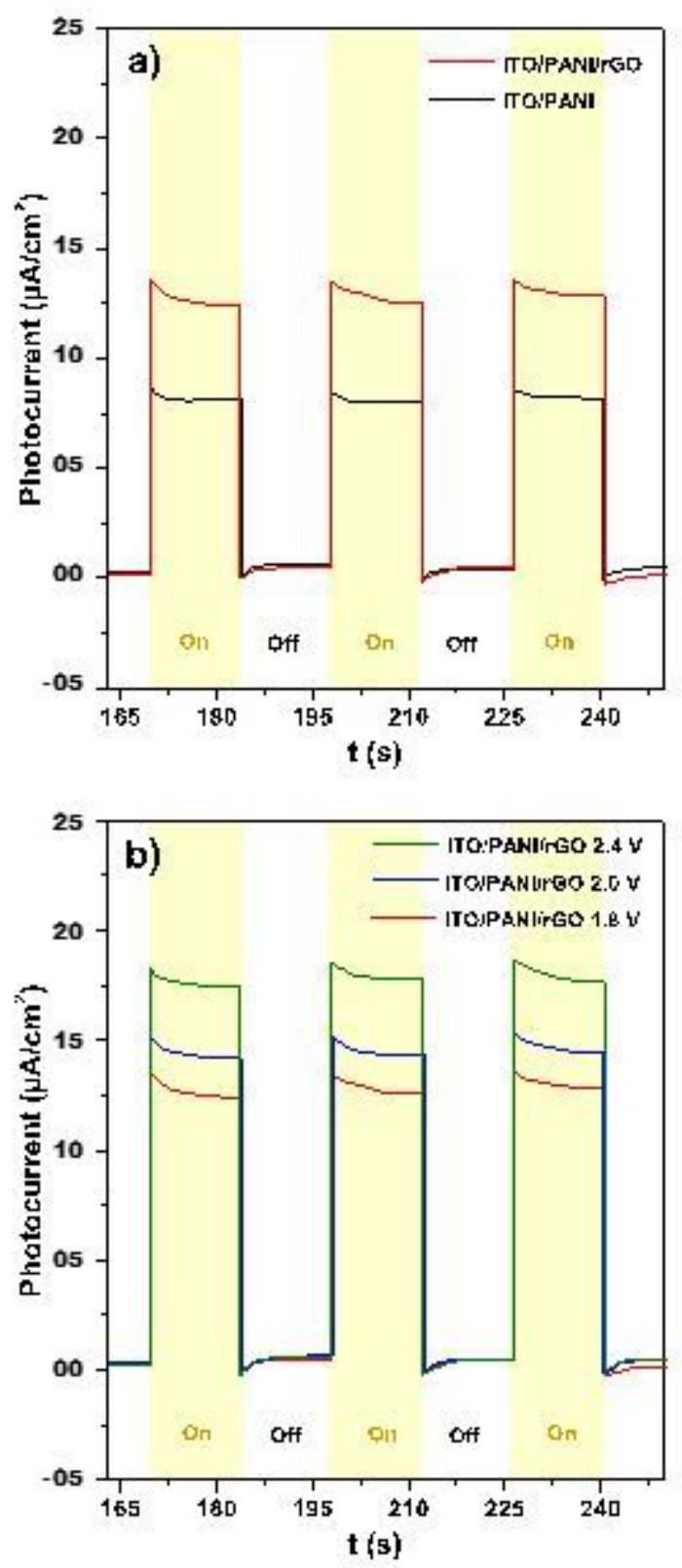

Figure 5

(a) Photocurrent response curves of ITO/PANI and ITO/PANI-rGO electrodes at a constant potential. (b) Photocurrent amplitude versus the applied potential for ITO/PANI-rGO. 

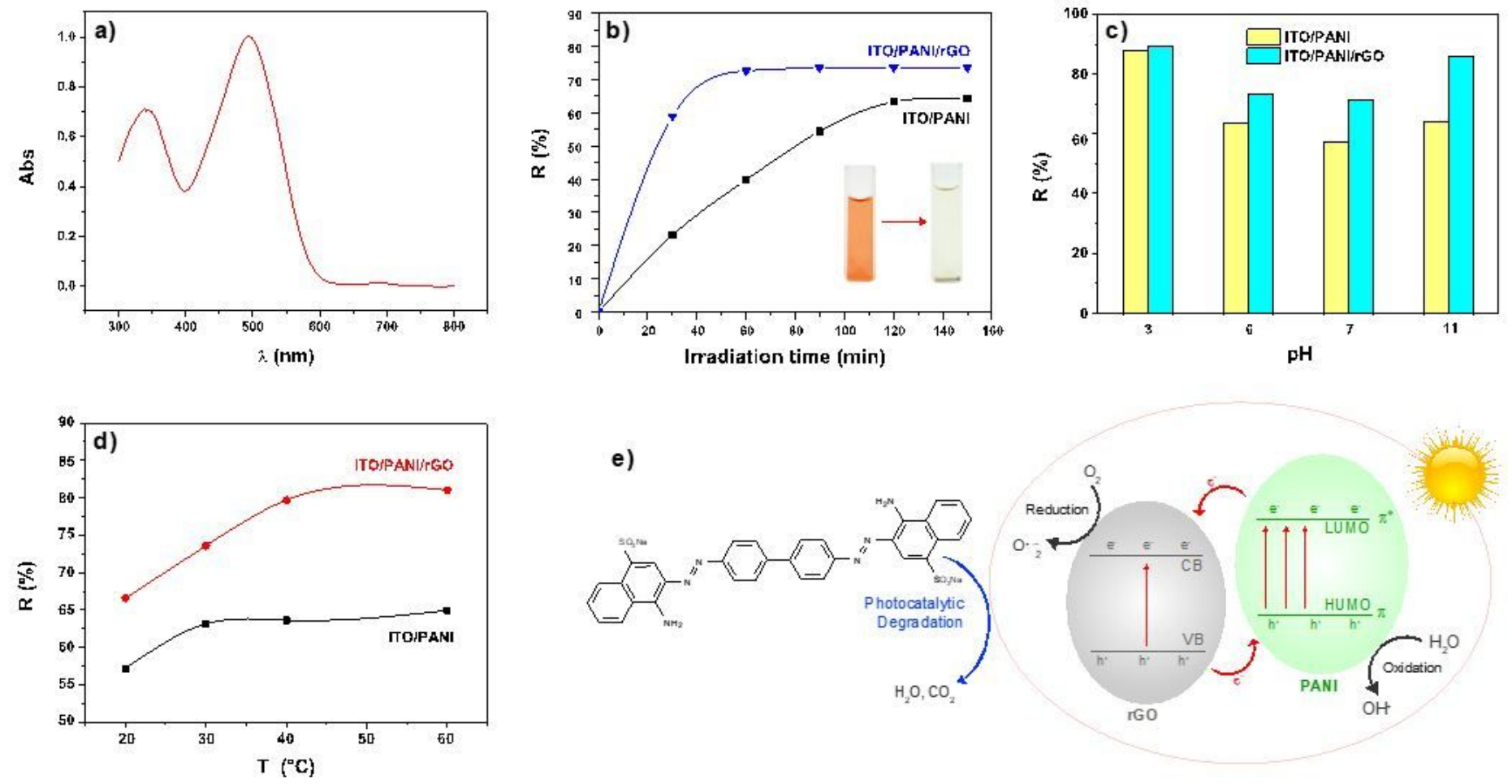

\section{Figure 6}

a) UV-Vis spectra of Congo Red. b) The photocatalytic degradation of Congo Red dye against specific time intervals under visible light irradiation. The $\mathrm{pH}$ effect (c) and temperature effect (d) on photodegradation rate. (e) Illustration suggested photocatalytic degradation of Congo Red by ITO/PANI-rGO thin films. 

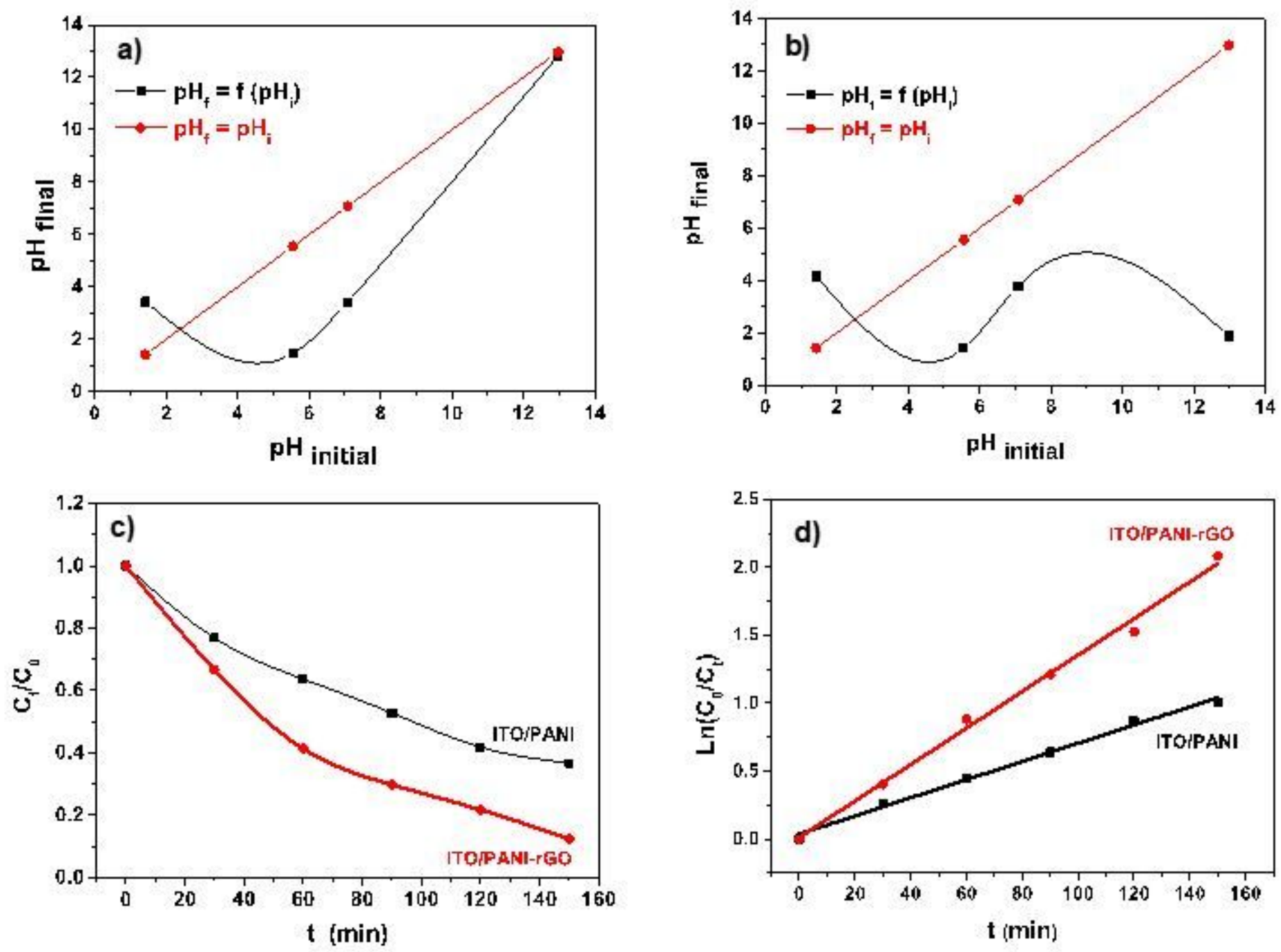

Figure 7

Curves of point of zero charge of ITO/PANI (a) and ITO/PANI/rGO (b) electrodes. Plot of (ct/c0) (c) and (b) In(c0/ct) (d) versus reaction time in the presence of the ITO/PANI and ITO/PANI-rGO nanocomposites. 

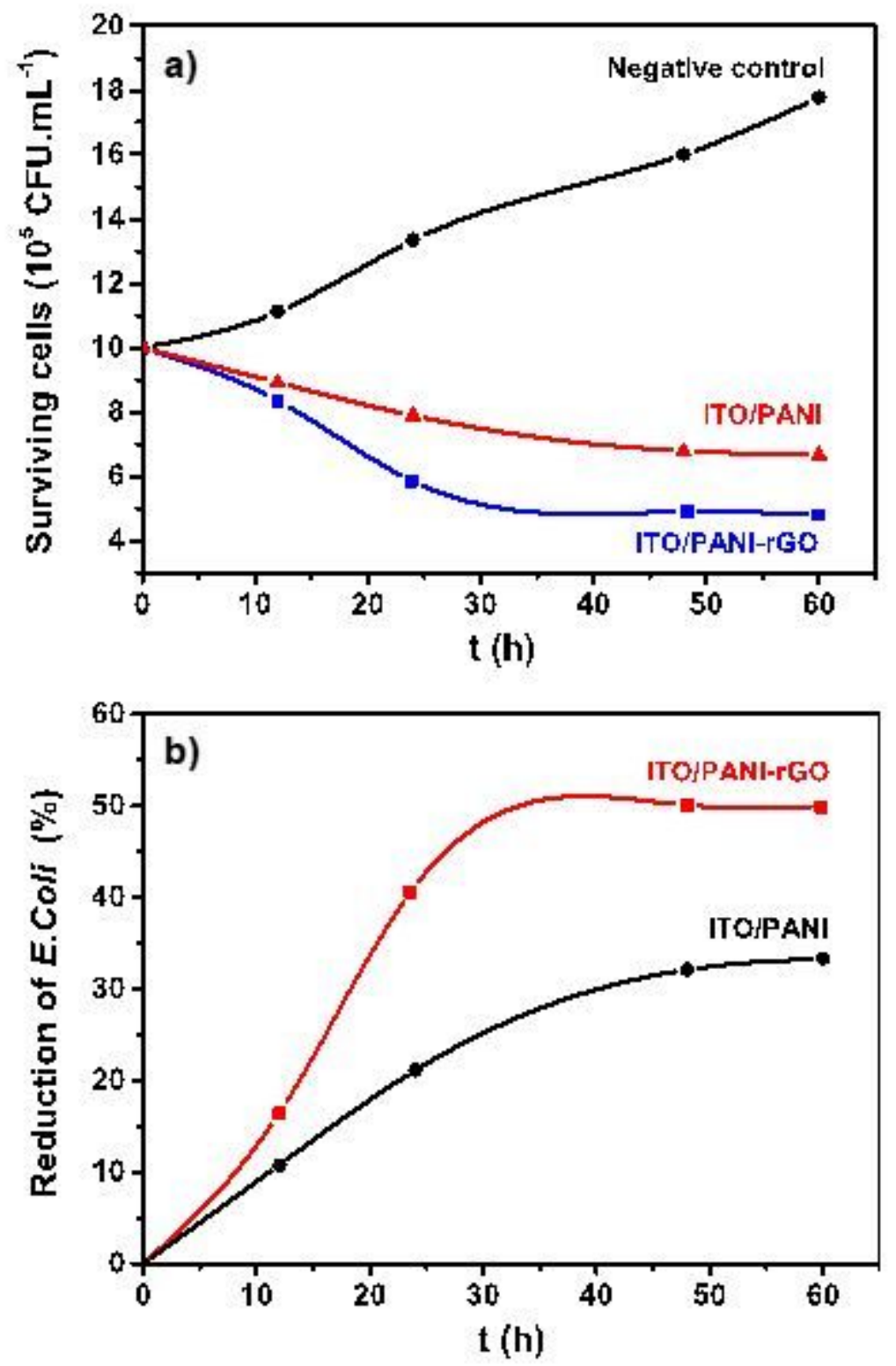

Figure 8

Antibacterial activities of the ITO/PANI and ITO/PANI-rGO nanocomposites

\section{Supplementary Files}

This is a list of supplementary files associated with this preprint. Click to download.

- GraphicalAbstract.docx

- Scheme1.docx 